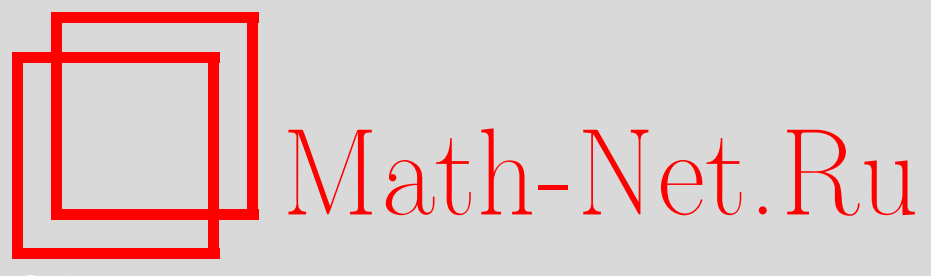

В. А. Ведерников, Н. В. Якубовский, Модули Шмидта и их некоторые применения, Матем. заметки, 2008, том 84, выпуск 5, 681-692

DOI: https://doi.org/10.4213/mzm4164

Использование Общероссийского математического портала Math-Net.Ru подразумевает, что вы прочитали и согласны с пользовательским соглашением http://www . mathnet.ru/rus/agreement

Параметры загрузки:

IP : 3.93 .64 .190

26 апреля 2023 г., 14:50:54

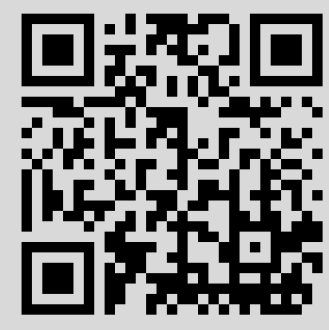


Том 84 выпуск 5 ноябрь 2008

УДК 512.553 .1

\section{Модули Шмидта и их некоторые применения}

\section{В. А. Ведерников, Н. В. Якубовский}

Пусть $R$ - ассоциативное кольцо с единицей. Неполупростой правый $R$-модуль $M=M_{R}$ назовем (правым) модулем Шмидта, если каждый собственный (правый) подмодуль из $M$ полупрост, и модуль $M$ назовем (правым) обобщенным модулем Шмидта, если $M$ не является модулем Шмидта, а каждый его собственный (правый) подмодуль либо полупрост, либо - модуль Шмидта. Аналогично определяется левый $R$-модуль Шмидта и левый обобщенный $R$-модуль Шмидта.

В работе получено полное описание строения правых $R$-модулей Шмидта и обобщенных $R$-модулей Шмидта, установлено существование подмодулей Шмидта в любом неполупростом артиновом модуле и дано полное описание неполупростых артиновых модулей, в которых каждый подмодуль Шмидта выделяется прямым слагаемым. В качестве следствий в работе получены характеризации (обобщенных) модулей Шмидта над Дедекиндовым кольцом и кольцом матриц над ним.

Библиография: 9 названий.

Пусть $\mathfrak{X}$ - непустой класс алгебр. При исследовании свойств $\mathfrak{X}$-алгебр существенную роль играют критические $\mathfrak{X}$-алгебры, т.е. $\mathfrak{X}$-алгебры, не обладающие некоторым свойством $\theta$, все собственные $\mathfrak{X}$-подалгебры которых этим свойством обладают. Так в работе [1] исследованы конечные ненильпотентные группы, все собственные подгруппы которых нильпотентны. Эти группы нашли многочисленные применения в теории групп и впоследствии были названы группами Шмидта (см. [2], [3]). Пусть $R$ - ассоциативное кольцо с единицей. Неполупростой правый $R$-модуль $M=M_{R}$ назовем (правым) модулем Шмидта, если каждый собственный (правый) подмодуль из $M$ полупрост и модуль $M$ назовем (правым) обобщенным модулем Шмид$m a$, если $M$ не является модулем Шмидта, а каждый его собственный (правый) подмодуль либо полупрост, либо - модуль Шмидта. Аналогично определяется левый $R$-модуль Шмидта и левый обобщенный $R$-модуль Шмидта. В работе показано, что классы модулей Шмидта и обобщенных модулей Шмидта непусты (см. пример 1 и примеры 2-4 соответственно) и различны (теорема 1 , следствие 1 и теорема 2).

Цель настоящей работы - дать полное описание строения правых $R$-модулей Шмидта и обобщенных $R$-модулей Шмидта, установить существование подмодулей Шмидта в любом неполупростом артиновом модуле и применить подмодули Шмидта к описанию строения некоторых артиновых модулей.

(C) В. А. Ведерников, Н. В. Якувовский, 2008 
Обозначения и определения, не приведенные в работе, можно найти в книгах [4][9]. В дальнейшем, если не оговорено противное, под $R$-модулем будем подразумевать правый $R$-модуль и применять следующие обозначения: через $A \leqslant M, A<M$ и $A<\cdot M$ будем обозначать соответственно: $A$ - подмодуль модуля $M, A$ - собственный подмодуль модуля $M$ и $A$ - максимальный подмодуль модуля $M$; через $A<^{b} M$ и $A<_{s} M$ будем обозначать соответственно: $A$ - существенный или большой (big) подмодуль модуля $M$ и $A$ - косущественный или малый (small) подмодуль модуля $M$. Собственный подмодуль $A$ модуля $M$ будем называть наибольшим (greatest) собственным подмодулем модуля $M$, если любой собственный подмодуль из $M$ содержится в $A$, и будем обозначать $A<^{g} M$. Под локалъным понимается модуль (не обязательно конечно порожденный), обладающий единственным максимальным подмодулем. Радикал модуля $M$, обозначаемый через $\operatorname{Rad}(M)$, определяется как пересечение всех максимальных подмодулей $M$, или, что то же самое, как сумма всех косущественных (малых) подмодулей $M$.

Лемма 1. Ненулевой модуль $M=M_{R}$ локален и иикличен тогда и только тогда, когда $\operatorname{Rad}(M)$ - наибольший собственный подмодуль в $M$.

ДокАЗАтЕЛЬСтво. Необходимость. Пусть $M=M_{R}-$ ненулевой циклический локальный модуль. Так как $M$ локален, то $\operatorname{Rad}(M)$ - единственный максимальный подмодуль в $M$. Поскольку $M$ цикличен, то по теореме 2.3 .11 из [4] каждый собственный подмодуль из $M$ лежит в $\operatorname{Rad}(M)$, т.е. $\operatorname{Rad}(M)$ - наибольший собственный подмодуль в $M$.

Достаточность. Пусть $M=M_{R}-$ модуль и $\operatorname{Rad}(M)$ - наибольший собственный подмодуль в $M$. Тогда $\operatorname{Rad}(M)$ - единственный максимальный подмодуль в $M$, т.е. $M$ - локальный модуль. Пусть $x$ принадлежит $M$ и $x \notin \operatorname{Rad}(M)$. Поскольку мы рассматриваем кольца с единицей, то $x R \not \subset \operatorname{Rad}(M)$. Отсюда следует, что $x R=M$, т.е. $M$ - циклический модуль. Лемма доказана.

ЗАмечаниЕ 1. Условие цикличности в лемме 1 является необходимым. Например, $\mathbb{Z}$-модуль $M=\mathbb{Q}_{\mathbb{Z}} \oplus\left(\mathbb{Z}_{2}\right)_{\mathbb{Z}}$ является локальным с единственным максимальным подмодулем $\operatorname{Rad}(M)=\mathbb{Q}_{\mathbb{Z}}$, который не является наибольшим собственным подмодулем в $M$.

ОПреДЕЛЕНИЕ 1. Неполупростой модуль $M=M_{R}$ назовем модулем Шмидта, если каждый его собственный подмодуль полупрост.

ПримеР 1. Модулем Шмидта является, модуль $M=\left(\mathbb{Z} / p^{2} \mathbb{Z}\right)_{\mathbb{Z}}$, где $p-$ простое число.

Лемма 2. Пусть $M=M_{R}$ - ненулевой модуль. Каждый собственный подмодуль в $M$ полупрост тогда и только тогда, когда выполняется одно из следующих условий:

1) $M$ - ненулевой полупростой модуль;

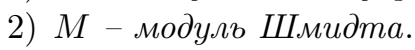

ДоказАТЕЛЬСтво. Необходимость. Пусть в ненулевом модуле $M=M_{R}$ каждый собственный подмодуль полупрост. Если $\operatorname{Soc}(M)=M$, то $M$ - полупростой модуль. Пусть $\operatorname{Soc}(M)<M$. Тогда $M$ - неполупростой модуль, а все собственные подмодули из $M$ полупросты и, значит, по определению $1 M$ - модуль Шмидта. 
Достаточность. Если $M=M_{R}$ - ненулевой полупростой модуль, то по следствию 8.1.5 из [4] каждый собственный подмодуль в $M$ полупрост. Если $M-$ модуль Шмидта, то по определению 1 каждый собственный подмодуль в $M$ полупрост. Лемма доказана.

Теорема 1. Для произвольного модуля $M=M_{R}$ следующие условия эквивалентны:

1) $М$ является модулем Шмидта;

2) $\operatorname{Soc}(M)$ - наибольший собственный подмодуль в $M$;

3) $M$ - неполупростой локальный ииклический модуль, $u \operatorname{Rad}(M)$ nолупрост.

ДокаЗАТЕЛЬСтво. 1) $\Rightarrow 2$ ). Пусть $M$ - модуль Шмидта. Тогда имеем следующее: $\operatorname{Soc}(M) \neq M$, каждый собственный подмодуль из $M$ полупрост и поэтому содержится в $\operatorname{Soc}(M)$. Следовательно, $\operatorname{Soc}(M)$ - наибольший собственный подмодуль в $M$.

$2) \Rightarrow 3)$ Так как $\operatorname{Soc}(M)$ - наибольший собственный подмодуль в $M$, то $\operatorname{Soc}(M)=$ $\operatorname{Rad}(M)$. Поэтому $\operatorname{Rad}(M)$ полупрост, а по лемме $1 M-$ локальный циклический модуль. При этом $M$ не является полупростым, так как $\operatorname{Soc}(M)<M$.

$3) \Rightarrow 1)$. Пусть $M$ - локальный циклический подмодуль, не являющийся полупростым, и $\operatorname{Rad}(M)$ - полупростой модуль. По лемме $1 \operatorname{Rad}(M)$ - наибольший собственный подмодуль в $M$. Тогда по следствию 8.1 .5 из [4] каждый собственный подмодуль в $M$ полупрост и по определению $1 M$ - модуль Шмидта. Теорема доказана.

СЛЕДСТвиЕ 1. Если $M-$ модуль Шмидта, то модуль $М$ не представим в виде суммы собственных подмодулей.

ДокАЗАТЕЛЬСтво. Так как по теореме 1 каждый собственный подмодуль модуля $M$ содержится в $\operatorname{Soc}(M)<^{g} M$, то сумма любой совокупности собственных подмодулей в $M$ содержится в $\operatorname{Soc}(M) \neq M$. Следствие доказано.

СлеДСтвиЕ 2. Если $M-$ модуль Шмидта, то имеем $\operatorname{Soc}(M)=\operatorname{Rad}(M) \neq 0$, $\operatorname{Soc}(M)<{ }_{s} M, \operatorname{Soc}(M)<\cdot M u \operatorname{Soc}(M)<{ }^{b} M$.

ДокАЗАТЕЛЬСтво. По теореме 1 Soc $(M)$ - наибольший собственный подмодуль в $M$. Поэтому $\operatorname{Soc}(M)$ - единственный максимальный подмодуль в $M$ и, значит, $\operatorname{Rad}(M)=\operatorname{Soc}(M)$. Так как $M-$ неполупростой модуль, а $M / \operatorname{Rad}(M)$ - простой модуль, то $\operatorname{Rad}(M) \neq 0$. Кроме того, из $\operatorname{Soc}(M)+U=M$ следует $U=M$ и из $\operatorname{Soc}(M) \cap U=0$ следует, что $U=0$. Поэтому $\operatorname{Soc}(M)<_{s} M$ и $\operatorname{Soc}(M)<^{b} M$. Следствие доказано.

СлЕДСТВИЕ 3. Любой ненулевой эпиморфный образ модуля Шмидта либо прост, либо является модулем Шмидта.

ДоКАЗАТЕЛЬСтво. Пусть $M=M_{R}$ - модуль Шмидта, $\varphi(M)=N$ - его ненулевой эпиморфный образ и $K=\operatorname{Ker}(\varphi)$. Тогда $M / K \cong N \neq 0$ и по теореме $1 K \leqslant \operatorname{Soc}(M)$. Если $K=\operatorname{Soc}(M)$, то в силу следствия $2 M / K$, а значит, и $N$ являются простыми модулями. Если $K<\operatorname{Soc}(M)=\operatorname{Rad}(M)$, то по теореме 9.2.1 (h) из [4] $\operatorname{Rad}(M) / K \leqslant$

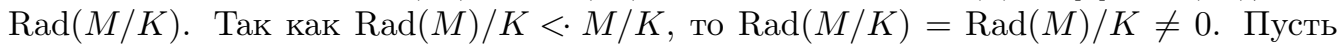


$L / K<M / K$. Тогда $L<M$ и, значит, по теореме $1 L \leqslant \operatorname{Soc}(M)$. Так как $L$ полупрост, то по следствию 8.1 .5 из [4] и $L / K$ полупрост. Тогда по лемме $2 M / K$, а значит, и $N$ являются модулями Шмидта. Следствие доказано.

Из теоремы 1 также можно вывести описание строения модулей Шмидта в некоторых конкретных случаях, в частности над дедекиндовыми кольцами. Вначале докажем вспомогательное

УтВеРЖДЕНИЕ. Произвольная область иелостности $R$ не содержит собственных минимальных идеалов.

ДоКАЗАтЕЛЬСтво. Пусть $R$ - область целостности, и пусть $I=x_{0} R$, где $x_{0} \in R$, $x_{0} \neq 0,-$ собственный главный идеал. Рассмотрим модули $R_{R}$ и $I_{R}$ и отображение $\varphi: R_{R} \rightarrow I_{R}$, заданное равенством $\varphi(r)=x_{0} r$. Легко проверяется, что $\varphi-$ модульный эпиморфизм. Пусть $r_{0} \in \operatorname{Ker} \varphi$. Тогда $\varphi\left(r_{0}\right)=x_{0} r_{0}=0$. Поскольку $R$ - область целостности, то $r_{0}=0$, откуда следует, что $\operatorname{Ker} \varphi=0$ и $I_{R} \cong R_{R}$. Теперь ясно, что модуль $I_{R}$ не может быть простым, т.е. идеал $I$ не может быть минимальным.

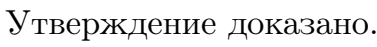

СлЕДСТВИЕ 4. Пусть $R$ - дедекиндово кольщо, не являющееся простым. Модуль $M=M_{R}$ является модулем Шмидта тогда и только тогда, когда $M \cong R / \mathfrak{p}^{2}$ для некоторого простого идеала $\mathfrak{p}$ колъца $R$.

ДоказАтельство. Необходимость. Пусть $R$ - дедекиндово кольцо, не являющееся простым кольцом, $M_{R}$ - модуль Шмидта. Так как по теореме $1 M_{R}$ конечно порожден, по утверждению 8.23 .9 (b) [7] $M_{R}=N_{R} \oplus\left(I_{1}\right)_{R} \oplus \cdots \oplus\left(I_{k}\right)_{R}$, где $N_{R}-$ периодический модуль, а $\left(I_{j}\right)$ - правый идеал в $R, j \in\{1,2, \ldots, k\}$. Так как по следствию $1 M_{R}$ неразложим, то $M_{R} \cong N_{R}$ или $M_{R} \cong I_{R}$ для некоторого $I \triangleleft R$. Однако во втором случае получаем, что модуль $R_{R}$ содержит собственный простой подмодуль (точнее - простой подмодуль из разложения $\left.\operatorname{Rad}(M)_{R}\right)$, т.е. $R$ содержит собственный минимальный идеал, что противоречит выше доказанному утверждению. Таким образом, получаем, что $M_{R}$ - периодический модуль. По предложению 23 [8; гл. VII, $\left.\S 5, \mathrm{n}^{\circ} 2\right]$ получаем (учитывая, что $M$ неразложим): $M \cong R / \mathfrak{p}^{n}$ для некоторого простого идеала $\mathfrak{p}$ кольца $R$. Так как $\operatorname{Rad}(M) \cong \mathfrak{p} / \mathfrak{p}^{n}$ и по теореме 1 $\operatorname{Rad}(M)$ - полупростой модуль, получаем, что $n=2$.

Достаточность очевидна.

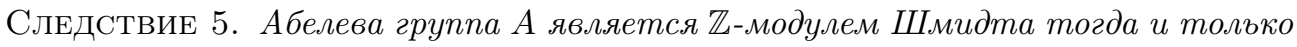
тогда, когда $A \cong \mathbb{Z}_{p^{2}}$, где $p$ - простое число.

ОПРЕДЕЛЕНИЕ 2. Неполупростой модуль $M=M_{R}$ назовем обобщеннъм модулем Шмидта, если $M$ не является модулем Шмидта, а каждый его собственный подмодуль либо полупрост, либо - модуль Шмидта.

Лемма 3. Пусть $M=M_{R}-$ обобщенный модуль Шмидта. Тогда выполняются следуюшие утверждения:

1) $М$ содержит подмодуль Шмидта, причем каждый подмодуль Шмидта максимален в $M$;

2) модуль $М$ конечно порожден;

3) среди максималъных подмодулей модуля $M$ не более одного полупростого подмодуля. 
ДокАзАтЕльство. 1) Так как $M$ не является модулем Шмидта, в силу определений 1 и 2 в $M$ существует собственный подмодуль Шмидта $A$. Допустим, что $A$ не является максимальным подмодулем в $M$. Тогда существует $B<M$ такой, что $A<B$. Так как $M$ - обобщенный модуль Шмидта, по определению $2 B$ полупрост или модуль Шмидта, и в любом случае $A$ полупрост, что противоречит определению 1. Следовательно, $A$ является максимальным подмодулем в $M$.

2) По пункту 1) $M$ содержит максимальный подмодуль Шмидта $A$. Пусть $x \in$ $M \backslash A$. Тогда $M=A+x R$. По теореме $1 A$ - циклический модуль. Тогда существует $a \in A$ такой, что $A=a R$ и $M=a R+x R$ конечно порожден.

3) Допустим, что среди максимальных подмодулей модуля $M$ имеется два различных полупростых подмодуля $C$ и $D$. Тогда $M=C+D$ и по следствию 8.1.5 из [4] модуль $M$ полупрост, что противоречит определению 2. Следовательно, среди максимальных подмодулей модуля $M$ не более одного полупростого подмодуля. Лемма доказана.

ЗАмечАниЕ 2. Применяя результаты леммы 3 , естественно классифицировать обобщенные модули Шмидта по количеству и типу их максимальных подмодулей.

Лемма 4. Пусть $M=M_{R}$ - обобщенный модуль Шмидта. Тогда выполняется одно из следующих утверждений:

1) $M$ содержит единственный максимальный подмодуль $K$, причем $K$ - модуль Шмидта. В этом случае назовем $M$ обобщенным модулем Шмидта 1-типа;

2) $M$ содержит максимальный подмодуль $P$, причем $P$ полупрост. $B$ этом случае назовем $M$ обобщенным модулем Шмидта 2-типа;

3) $М$ содержит более одного максимального подмодуля, причем каждый из них является модулем Шмидта. В этом случае назовем $M$ обобщенным модулем Шмидта 3-типа.

ДокАЗАтЕЛЬСтво. Пусть $M=M_{R}$ - обобщенный модуль Шмидта. Тогда по лемме $4 M$ содержит максимальный подмодуль Шмидта $K$. Если $M$ локален, то выполняется утверждение 1). Пусть $M$ не является локальным. По лемме 4 среди максимальных подмодулей модуля $M$ не более одного полупростого подмодуля. Если $M$ содержит максимальный подмодуль $P$ такой, что $P$ полупрост, то выполняется утверждение 2). Если $M$ не содержит максимальный полупростой подмодуль, то выполняется утверждение 3). Лемма доказана.

Укажем конкретные примеры обобщенных модулей Шмидта различных типов.

ПримеР 2. Модуль $M=\left(\mathbb{Z} / p^{3} \mathbb{Z}\right)_{\mathbb{Z}}$, где $p$ - простое число, является обобщенным модулем Шмидта 1-типа.

ПримеР 3. Модуль $M=\left(\mathbb{Z} / p^{2} \mathbb{Z}\right)_{\mathbb{Z}} \oplus(\mathbb{Z} / q \mathbb{Z})_{\mathbb{Z}}$, где $p$ и $q$ - простые числа, является обобщенным модулем Шмидта 2-типа. Заметим, что не обязательно $p \neq q$.

ПримеР 4. Рассмотрим матричное кольцо

$$
R=\left\{\left(\begin{array}{ll}
a & b \\
0 & c
\end{array}\right) \mid a \in F_{2}, b, c \in F_{4}\right\}
$$


где $F_{q}$ - поле Галуа из $q$ элементов, причем $F_{4}=\{0,1, \alpha, 1+\alpha\}$. Теперь возьмем правый $R$-модуль

$$
M=\left\{\left(\begin{array}{ll}
x & y \\
0 & 0
\end{array}\right) \mid x, y \in F_{4}\right\} .
$$

Покажем, что $M$ является обобщенным модулем Шмидта 3-типа.

Перечислим все его собственные подмодули. Множество элементов

$$
K=\left\{\left(\begin{array}{ll}
x & 0 \\
0 & 0
\end{array}\right) \mid x \in F_{4}\right\}
$$

не является подмодулем в $M$. Действительно, если $x_{0} \neq 0$, то

$$
\left(\begin{array}{cc}
x_{0} & 0 \\
0 & 0
\end{array}\right) \cdot\left(\begin{array}{ll}
0 & 1 \\
0 & 0
\end{array}\right)=\left(\begin{array}{cc}
0 & x_{0} \\
0 & 0
\end{array}\right) \notin K .
$$

Пусть $D$ - ненулевой подмодуль в $M$. Тогда $D$ содержит матрицу с ненулевым вторым столбцом. Пусть

$$
d=\left(\begin{array}{ll}
* & \gamma \\
0 & 0
\end{array}\right) \in D, \quad \gamma \neq 0
$$

Тогда для любого $x \in F_{4}$ существует $\delta \in F_{4}$ такое, что $x=\delta \gamma$. Пусть

$$
r=\left(\begin{array}{ll}
1 & 0 \\
0 & \delta
\end{array}\right) \in R .
$$

Тогда

$$
d r=\left(\begin{array}{ll}
* & x \\
0 & 0
\end{array}\right) \in D .
$$

Пусть $D_{i j}$ - множество всех элементов из $F_{4}$, встречающихся в матрицах из $D$ в $i$-й строке в $j$-м столбце. Следовательно, $D_{12}=F_{4}$. Поэтому подмодуль

$$
N=\left\{\left(\begin{array}{ll}
0 & y \\
0 & 0
\end{array}\right) \mid y \in F_{4}\right\}
$$

содержится в $D$. Так как $N$ порождается любым своим ненулевым элементом, то $N$ является простым подмодулем в $M$. Если $D_{11}=\{0\}$, то $D=N$. При $D_{11}=F_{4}$ получим $D=M$. Так как $D_{11}$ - подгруппа аддитивной группы $F_{4}$, то для $D_{11}$ имеются еще лишь следующие возможности:

1) $D_{11}=\{0,1\}$; тогда $D=M_{1}$ и $N<M_{1}<M$;

2) $D_{11}=\{0, \alpha\}$; тогда $D=M_{2}$ и $N<M_{2}<M$;

3) $D_{11}=\{0,1+\alpha\}$; тогда $D=M_{3}$ и $N<M_{3}<M$.

Ясно, что $M_{i}$ является максимальным подмодулем в $M$. Так как $N$ содержится в каждом ненулевом подмодуле из $M$, то $M_{i}$ не является полупростым модулем, а из $\left|M_{i}\right|=8$ и $|N|=4$ следует, что $N$ - единственный максимальный подмодуль из $M_{i}$ для всех $i \in\{1,2,3\}$. Следовательно, $M_{i}$ - модуль Шмидта для всех $i \in\{1,2,3\}$, а $M$ - обобщенный модуль Шмидта 3 -типа.

ТеОрема 2. Модуль $M=M_{R}$ является обобщенным модулем Шмидта тогда и только тогда, когда выполняется одно их следующих утверждении: 
1) $M$ - локальный циклический модуль и $\operatorname{Rad}(M)$ - модуль Шмидта;

2) $M=S \oplus P$, где $S$ - модуль Шмидта, $P$ - простой модулъ;

3) $M=S_{1}+S_{2}$, где $S_{1}$ и $S_{2}-$ подмодули Шмидта, $S_{1} \cap S_{2}=\operatorname{Rad}(M)$ и каждъй подмодуль из $M$, не содержащийся в $\operatorname{Rad}(M)$, неразложим.

ДокАЗАТЕЛЬСтво. Необходимость. Пусть $M$ - обобщенный модуль Шмидта. По лемме $4 M$ конечно порожден и содержит подмодуль Шмидта, причем каждый подмодуль Шмидта максимален в $M$.

Пусть $M$ - обобщенный модуль Шмидта 1 -типа. Тогда по лемме $4 \operatorname{Rad}(M)<\cdot M-$ модуль Шмидта, и $M$ локален. Так как $M$ локален и конечно порожден, отсюда следует, что $M$ цикличен. Следовательно, выполняется утверждение 1).

Пусть $M$ - обобщенный модуль Шмидта 2-типа, $N$ и $S$ - максимальные подмодули в $M$, причем $N$ - полупростой и $S$ - подмодуль Шмидта модуля $M$. Пусть $D=S \cap N$. Так как $N$ полупрост, существует $P<N$ такой, что $N=D \oplus P$. Тогда $M=S+N=$ $S+(D \oplus P)=S \oplus P$. Так как $S<\cdot M$, то $P \cong M / S-$ простой модуль. Следовательно, выполняется утверждение 2).

Пусть $M$ - обобщенный модуль Шмидта 3-типа. По лемме $4 M$ содержит более одного максимального подмодуля, причем каждый из них является модулем Шмидта. Пусть $S_{1}$ и $S_{2}$ - различные максимальные подмодули Шмидта модуля $M$. Тогда $M=S_{1}+S_{2}$. По теореме 1 модуль Шмидта циклический, поэтому существуют $a_{i} \in S_{i}$ такие, что $S_{i}=a_{i} R,(i=1,2)$. Тогда $M=a_{1} R+a_{2} R$ и, значит, модуль $M$ конечно порожден. Так как $M / S_{2} \cong S_{1} / S_{1} \cap S_{2}$ - простой модуль, по следствию $2 \operatorname{Rad}\left(S_{1}\right)=\operatorname{Soc}\left(S_{1}\right)=S_{1} \cap S_{2} \supseteq \operatorname{Rad}(M)$. По следствию 9.1 .5 из [4] $\operatorname{Rad}\left(S_{1}\right) \subseteq \operatorname{Rad}(M)$. Значит, $\operatorname{Rad}(M)=\operatorname{Rad}\left(S_{1}\right)=S_{1} \cap S_{2}$. Аналогично получим, что $\operatorname{Rad}(H)=\operatorname{Rad}(M)$ для любого подмодуля Шмидта $H$ модуля $M$.

Пусть $N \leqslant M$ и $N \nsubseteq \operatorname{Rad}(M)$. Покажем, что $N$ - неразложимый модуль. Пусть $N<M$. Так как $M$ конечно порожден, по теореме 2.3.11 из [4] $N$ содержится в некотором максимальном подмодуле $S$ модуля $M$. Поскольку $S$ является модулем Шмидта, то $\operatorname{Rad}(M)=\operatorname{Rad}(S)$. Так как $N \nsubseteq \operatorname{Rad}(S)$, по теореме 1 получим, что $N=S$ и, значит, по следствию $1 N$ - неразложимый модуль. Пусть $N=M$. Допустим, что $M=X \oplus Y$, где $X<M$ и $Y<M$. Пусть $X \leqslant A<\cdot M$. Так как $A$ - модуль Шмидта, и по модулярному тождеству $A=X+(A \cap Y)$, по следствию 1 получим, что $A=X<\cdot M$. Аналогично $Y<\cdot M$. Тогда $\operatorname{Rad}(M) \subseteq X \cap Y=0$, что невозможно. Следовательно, $M$ - неразложимый модуль.

Достаточность. Пусть $M-$ модуль из пункта 1$)$ теоремы. Тогда $\operatorname{Rad}(M)-$ наибольший собственный подмодуль в $M$. Пусть $N<M$. Тогда $N<\operatorname{Rad}(M)$ или $N=\operatorname{Rad}(M)$, т.е. всякий подмодуль в $M$ либо полупрост, либо - модуль Шмидта. Значит, по определению $2 M$ - обобщенный модуль Шмидта, причем по лемме 4 $M$ - обобщенный модуль Шмидта 1-типа.

Пусть $M$ - модуль из пункта 2) теоремы. Тогда $M=S \oplus P$, где $S$ - модуль Шмидта, $P$ - простой модуль. Так как $M$ конечно порожден, существует $L<\cdot M$ такой, что $P<L$. По модулярному тождеству Дедекинда имеем $L=P \oplus(L \cap S)$, причем $L \cap S$ полупрост, поэтому $L$ также полупрост. Предположим теперь, что $N<\cdot M$ и $P \not \subset N$. Ясно, что $N \cap P=0$ и $M=N \oplus P$. Тогда $N \cong M / P \cong S$ и, значит, $N-$ модуль Шмидта. Таким образом, каждый максимальный подмодуль в $M$ либо полупрост, либо - модуль Шмидта. Так как $M$ конечно порожден, каждый его собственный 
подмодуль содержится в некотором максимальном подмодуле. Следовательно, $M-$ обобщенный модуль Шмидта 2-типа.

Пусть $M$ - модуль из пункта 3) теоремы. Так как $M=S_{1}+S_{2}$, где $S_{1}$ и $S_{2}$ подмодули Шмидта, то $M$ конечно порожден. Из $S_{1} \cap S_{2}=\operatorname{Rad}(M)$ по теореме 1 следует, что $\operatorname{Rad}(M) \neq 0$ полупрост.

Пусть $S<\cdot M$. Покажем, что $S$ является модулем Шмидта. Поскольку каждый подмодуль из $M$, не содержащийся в $\operatorname{Rad}(M)$, неразложим и $\operatorname{Rad}(M)<S$, то $S$ неполупрост. Можем считать, что $S \neq S_{1}$ и $S \neq S_{2}$. Тогда из $M=S+S_{2}$ следует, что $M / S_{2} \cong S / S \cap S_{2}$ - простой модуль и, значит, $S \cap S_{2}=\operatorname{Soc}(S)<\cdot S$. Допустим, что в $S$ существует собственный подмодуль $L$ такой, что $L \nsubseteq \operatorname{Soc}(S)$. Тогда $S=L+\operatorname{Soc}(S)$. Пусть $D=L \cap \operatorname{Soc}(S)$ и $\operatorname{Soc}(S)=D \oplus K$. Тогда $S=L \oplus K$, что невозможно. Следовательно, $\operatorname{Soc}(S)$ - наибольший собственный подмодуль в $S$ и по теореме $1 S-$ модуль Шмидта. Поэтому в модуле $M$ каждый максимальный подмодуль является модулем Шмидта и, значит, $M$ - обобщенный модуль Шмидта 3-типа. Теорема доказана.

СЛЕДСТВИЕ 6. В ненулевом конечно порожденном модуле $M=M_{R}$ каждъй максимальный подмодуль является модулем Шмидта тогда и только тогда, когда M - обобщенный модуль Шмидта 1-типа или 3-типа.

ДокАЗАтеЛьство. Достаточность вытекает из лемм 3 и 4.

Необходимость. Пусть в ненулевом конечно порожденном модуле $M=M_{R}$ каждый максимальный подмодуль является модулем Шмидта. Ясно, что $M-$ неполупростой модуль и не является модулем Шмидта. Пусть $N$ - произвольный собственный подмодуль в $M$. По теореме 2.3.11 [4] существует $A<\cdot M$ такой, что $N \leqslant A$. Таким образом, в $M$ каждый собственный подмодуль либо полупрост, либо - модуль Шмидта, и согласно определению $2 M$ - обобщенный модуль Шмидта. По лемме 4 $M$ - обобщенный модуль Шмидта 1-типа или 3-типа. Следствие доказано.

ЗАмЕчАниЕ 3. Представляется интересным получить описание строения конечно порожденных $R$-модулей, в которых каждый второй (третий, $n$-й) максимальный подмодуль является модулем Шмидта.

Используя теорему 2 и следствие 4, получим описание строения обобщенных модулей Шмидта над дедекиндовыми кольцами.

СлеДСТВИЕ 7. Пусть $R$ - дедекиндово кольщо, не являющееся простым. Модуль $M=M_{R}$ является обобщенным модулем Шмидта тогда и только тогда, когда выполняется одно из следующих утверждений:

1) $M \cong R / \mathfrak{p}^{3}$, для некоторого простого идеала $\mathfrak{p}$ кольца $R$;

2) $M \cong R / \mathfrak{p}^{2} \oplus R / \mathfrak{q}$ для некоторых простых (не обязательно различных) идеалов $\mathfrak{p} u \mathfrak{q}$ кольиа $R$.

ДоКАЗАтЕЛьСтво. Пусть $M_{R}$ - обобщенный модуль Шмидта; тогда, используя лемму 3,2 ) и утверждение 8.23 .9 (b) [7], получаем, что $M_{R}=N_{R} \oplus\left(I_{1}\right)_{R} \oplus \cdots \oplus\left(I_{k}\right)_{R}$, где $N_{R}$ - периодический модуль, а $\left(I_{j}\right)$ - правый идеал в $R, j \in\{1,2, \ldots, k\}$. Пусть $M_{R}$ - обобщенный модуль Шмидта 1 -типа или 3 -типа. Используя тот факт, что $M_{R}$ неразложим (теорема 2), и рассуждая точно так же, как при доказательстве следствия 4 , получаем что $M_{R}$ - периодический модуль. Пусть $M_{R}$ - обобщенный 
модуль Шмидта 2-типа. Тогда $M_{R}=S_{R} \oplus P_{R}$, где $S_{R}$ - модуль Шмидта, $P_{R}-$ простой модуль. Как было показано выше, ни $S_{R}$, ни $P_{R}$ не могут быть изоморфны идеалу кольца $R$, откуда также следует, что $M_{R}$ - периодический модуль.

По предложению 23 [8; гл. VII, §5, $\left.\mathrm{n}^{\circ} 2\right]$ получаем, что $M \cong \bigoplus_{i \in I}\left(R / \mathfrak{p}_{i}^{n_{i}}\right)$ для некоторых конечных семейств $\left(n_{i}\right)_{i \in I}$ и $\left(\mathfrak{p}_{i}\right)_{i \in I}$, где $n_{i}$ - натуральные числа, а $\mathfrak{p}_{i}-$ простые идеалы кольца $R$. Если $M$ - обобщенный модуль Шмидта 1-типа, то он локален и цикличен по теореме 2 ; в этом случае $I$ состоит из одного элемента и $M \cong$ $R / \mathfrak{p}^{n}$. Ясно, что $\operatorname{Rad}(M) \cong \mathfrak{p} / \mathfrak{p}^{n}$. Так как по теореме $2 \operatorname{Rad}(M)$ - модуль Шмидта, то $\operatorname{Rad}(\operatorname{Rad}(M)) \cong \mathfrak{p}^{2} / \mathfrak{p}^{n}-$ полупростой модуль, откуда $n=3$. Пусть $M$ - обобщенный модуль Шмидта 2-типа. Тогда по теореме $2 M$ является прямой суммой некоторого модуля Шмидта и простого модуля. Значит, $I$ содержит два элемента, и $M \cong$ $R / \mathfrak{p}^{n_{1}} \oplus R / \mathfrak{q}^{n_{2}}$, где $\mathfrak{p}$ и $\mathfrak{q}$ не обязательно различны. Положив для определенности, что $R / \mathfrak{q}^{n_{2}}-$ простой модуль, и используя следствие 4 , получим, что $n_{1}=2$ и $n_{2}=1$. Предположив, что $M$ - обобщенный модуль Шмидта 3 -типа, получим противоречие. Действительно, по теореме $2 M$ не локален, и поэтому $I$ содержит более одного элемента. Но тогда $M$ разложим - противоречие с теоремой 2.

Достаточность. Пусть $M=M_{R}$, где $R$ - дедекиндово кольцо, не являющееся простым и $M \cong R / \mathfrak{p}^{3}$ для некоторого простого идеала $\mathfrak{p}$ кольца $R$. Ясно, что $\operatorname{Rad}(M) \cong \mathfrak{p} / \mathfrak{p}^{3}$, а значит, $M$ - локальный циклический модуль и $\operatorname{Rad}(M)$ - модуль Шмидта. По теореме $2 M$ - обобщенный модуль Шмидта 1-типа. Если же $M \cong R / \mathfrak{p}^{2} \oplus R / \mathfrak{q}$ для некоторых простых (не обязательно различных) идеалов $\mathfrak{p}$ и $\mathfrak{q}$ кольца $R$, то по следствию $4 M$ есть прямая сумма модуля Шмидта и простого модуля; значит, $M$ - обобщенный модуль Шмидта 2-типа. Следствие доказано.

СлеДСтвиЕ 8. Абелева группа А является обобщенным $\mathbb{Z}$-модулем Шмидта тогда и только тогда, когда выполняется одно из следующих утверждений:

1) $A \cong \mathbb{Z}_{p^{3}}$, где $p$ - простое число;

2) $A \cong \mathbb{Z}_{p^{2}} \oplus \mathbb{Z}_{q}$, где р и $q$ - простые (не обязательно) различные числа.

Покажем, что модули Шмидта достаточно распространены, точнее

Лемма 5. Если $M=M_{R}-$ неполупростой артинов модуль, то $M$ содержит подмодуль Шмидта.

ДокАЗАТЕЛЬСтво. Пусть $M=M_{R}$ - неполупростой артинов модуль. Если в $M$ все подмодули полупросты, то по определению $1 M$ - модуль Шмидта и доказывать нечего. Пусть $M$ содержит собственный неполупростой подмодуль $M_{1}<M$. Если в $M_{1}$ все собственные подмодули полупросты, то по определению $1 M_{1}$ - модуль Шмидта и лемма доказана. Пусть $M_{1}$ содержит собственный неполупростой подмодуль $M_{2}$. Продолжая этот процесс, получим $M>M_{1}>M_{2}>\cdots$. Так как $M$ артинов модуль, через конечное число шагов найдется неполупростой модуль $M_{n}$, все собственные подмодули которого полупросты. Следовательно, $M_{n}$ - подмодуль Шмидта модуля $M$. Лемма доказана.

Итак, мы показали, что каждый неполупростой артинов модуль содержит подмодуль Шмидта. Наличие определенных свойств у подмодулей Шмидта в неполупростом артиновом модуле $M$ позволяет его достаточно точно описать. 
Теорема 3. В неполупростом артиновом модуле $M=M_{R}$ каждый подмодуль Шмидта выделяется прямым слагаемым тогда и толъко тогда, когда

$$
M=S_{1} \oplus \cdots \oplus S_{k} \oplus P_{1} \oplus \cdots \oplus P_{l},
$$

где $S_{i}, i=1, \ldots, k,-$ артинов подмодуль Шмидта и $P_{j}, j=1, \ldots, l,-$ простой подмодуль модуля $M$, причем для каждого подмодуля Шмидта $S$ модуля $M$ существует индекс $r \in\{1,2, \ldots, k\}$ такой, что $S \cong S_{r} u$

$$
M=S_{1} \oplus \cdots \oplus S_{r-1} \oplus S \oplus S_{r+1} \oplus \cdots \oplus S_{k} \oplus P_{1} \oplus \cdots \oplus P_{l} .
$$

ДоказАтельство. Необходимость. По лемме $5 M$ содержит подмодуль Шмидта $S_{1}$. Тогда $M=S_{1} \oplus M_{1}$. Заметим, что здесь оба подмодуля артиновы, так как артинов модуль $M$. Если при этом $M_{1}$ - полупростой модуль, то он разлагается в прямую сумму конечного числа простых модулей и мы получаем искомое разложение модуля $M$. Пусть $M_{1}$ не является полупростым. Тогда по лемме 5 в $M_{1}$ существует подмодуль Шмидта $S_{2}$. Так как $S_{2}$ выделяется в $M$ прямым слагаемым, в $M$ найдется подмодуль $N$ такой, что $M=S_{2} \oplus N$. Тогда по модулярному тождеству получим, что $M_{1}=S_{2} \oplus M_{2}$, где $M_{2}=M_{1} \cap N$ и, значит, $M=S_{1} \oplus S_{2} \oplus M_{2}$, причем $M>M_{1}>M_{2}$. Так как $M$ - артинов модуль, продолжая этот процесс, через конечное число шагов получим, что $M=S_{1} \oplus S_{2} \oplus \cdots \oplus S_{k} \oplus P$, где $P$ - полупростой подмодуль модуля $M$. Так как $P$ артинов, он разлагается в прямую сумму конечного числа простых модулей $P_{1}, \ldots, P_{l}$ и мы получаем для $M$ разложение (1). Заметим, что $M$ - модуль конечной длины. Действительно, пусть $S_{i}$ - произвольный модуль Шмидта из указанного разложения. Так как $S_{i}-$ артинов модуль, то $\operatorname{Soc}\left(S_{i}\right)$ также артинов. По теореме 8.1.6 [4] $\operatorname{Soc}\left(S_{i}\right)$ - модуль конечной длины. Так как по следствию $2 \operatorname{Soc}\left(S_{i}\right)<\cdot S_{i}$, то $S_{i}$ - модуль конечной длины. Поэтому, по следствию 6.1 .3 из [4] $M-$ модуль конечной длины.

Пусть теперь $S$ - произвольный подмодуль Шмидта модуля $M$. По условию $M=$ $S \oplus T$, где $T<M$. Как и выше, нетрудно построить для модуля $M$ разложение (2) вида

$$
M=F_{1} \oplus \cdots \oplus F_{t} \oplus Q_{1} \oplus \cdots \oplus Q_{s},
$$

где $F_{i}, i=1, \ldots, t,-$ подмодуль Шмидта, $F_{1}=S$ и $Q_{j}, j=1, \ldots, s,-$ простой подмодуль модуля $M$. По теореме Шмидта-Орэ (теорема IV.3.12 из [9]) имеем следующее: $k=t, l=s$ и существует индекс $r \in\{1,2, \ldots, k\}$ такой, что $S \cong S_{r}$ и $M=S_{1} \oplus \cdots \oplus S_{r-1} \oplus S \oplus S_{r+1} \oplus \cdots \oplus S_{k} \oplus P_{1} \oplus \cdots \oplus P_{l}$.

Достаточность очевидна. Теорема доказана.

СЛЕДСТВИЕ 9. Если в неполупростом артиновом модуле $M=M_{R}$ каждый подмодуль Шмидта выделяется прямым слагаемым, то $M$ - модуль конечной длины.

ДокАЗАТЕЛЬСтво содержится в тексте теоремы 3.

Замечание 4 . Напомним, что кольца $A$ и $B$ называются подобными (или эквивалентными в смысле Мориты), если эквивалентны категории $\bmod -A$ и mod- $B$. Говорят, что категории $\mathfrak{C}$ и $\mathfrak{D}$ эквивалентны, если существуют такие функторы $T: \mathfrak{C} \rightsquigarrow \mathfrak{D}$ и $S: \mathfrak{D} \rightsquigarrow \mathfrak{C}$, что функторы $T S$ и $S T$ естественно изоморфны тождественным функторам $\operatorname{Id}_{\mathfrak{D}}$ и $\operatorname{Id}_{\mathfrak{C}}$ соответственно [6; т. 2, с. 402], и кратко обозначают в этом случае $T: \mathfrak{C} \rightsquigarrow \mathfrak{D}$ - эквивалентность [7; с. 97]. 
Теорема 4. Пусть кольиа $A$ и В эквивалентны в смысле Мориты, и пустъ $T: \bmod -A \rightsquigarrow \bmod -B$ - соответствующая эквивалентность категорий $\bmod -A u$ mod-B. Тогда справедливы следующие утверждения:

1) если $M=M_{A}-$ модуль Шмидта, то ТM $=(T M)_{B}-$ также модуль Шмид$m a$

1) если $M=M_{A}$ - обобщенный модуль Шмидта i-muпа, то $T M=(T M)_{B}-$ также обобщенный модуль Шмидта $i$-типа, $i=1,2,3$.

ДокАзАтельство. 1) Так как эквивалентность $T$ переводит полупростые $A$-модули в полупростые $B$-модули (см. 4.33 в [7]) и, кроме того, существует обратная эквивалентность $T^{-1}$, то модуль $T M$ не является полупростым, а все его собственные подмодули полупросты. По лемме $2 T M$ - модуль Шмидта.

2) Пусть $M$ - обобщенный $A$-модуль Шмидта. Учитывая предыдущее замечание, получаем, что $B$-модуль $T M$ не является ни полупростым, ни модулем Шмидта. Так как $T$ переводит максимальные подмодули $A$-модуля $M$ в максимальные подмодули $B$-модуля $T M$ и, кроме того, существует обратная эквивалентность $T^{-1}$, используя определение 2 и лемму 4 , получим, что обобщенный $A$-модуль Шмидта $i$-типа $M$ переходит в обобщенный $B$-модуль Шмидта $i$-типа $T M$. Теорема доказана.

СЛЕДСТВИЕ 10. Пусть $R$ - дедекиндово колъцо, $R_{n}$ - полное кольцо матрии, над $R$. Тогда справедливы следующие утверждения:

1) модуль $M=M_{R_{n}}$ является модулем Шмидта тогда и только тогда, когда $M-$ чепной модуль длины 2 ;

2) модуль $M=M_{R_{n}}$ является обобщенным модулем Шмидта 1-типа тогда и только тогда, когда $M$ - цепной модуль длинъ 3 ;

3) модуль $M=M_{R_{n}}$ является обобщенным модулем Шмидта 2-типа тогда и только тогда, когда $M=S \oplus P$, где $S$ - цепной модуль длины 2 , а $P$ простой модуль.

ДоказАтеЛЬСтво. Кольцо $R$ эквивалентно в смысле Мориты кольцу $R_{n}[6 ;$ т. 1 , с. 530]. Поэтому справедливость утверждения непосредственно вытекает из теоремы 4 , следствий 4 и 7 . Следствие доказано.

ЗАмЕчАниЕ 5. Для индуктивного построения новых классов конечно порожденных $R$-модулей введем следующие обозначения: $\mathfrak{S}_{0}-$ класс всех $R$-модулей Шмидта; $\mathfrak{S}_{1}-$ класс всех $R$-модулей, в которых каждый максимальный подмодуль является модулем Шмидта, т.е. принадлежит $\mathfrak{S}_{0}$, и $\mathfrak{S}_{n+1}-$ класс всех $R$-модулей, в которых каждый максимальный подмодуль принадлежит $\mathfrak{S}_{n}$ для любого $n \in \mathbb{N}$. Отметим, что $R$-модули из класса $\mathfrak{S}_{1}$ являются обобщенными $R$-модулями Шмидта (1-типа или 3 -типа). Возникает вопрос: "не будет ли последовательность классов $R$-модулей

$$
\mathfrak{S}_{0}, \mathfrak{S}_{1}, \ldots, \mathfrak{S}_{n}, \ldots
$$

стабилизироваться на конечном шаге?" Для произвольного ассоциативного кольца $R$ с единицей это неверно. Действительно, существует аддитивная циклическая $p$-группа $P_{n}$ порядка $p^{n+2}$ для любого $n \in \mathbb{N}$. Рассматривая $P_{n}$, как $\mathbb{Z}$-модуль, замечаем, что $P_{n} \in \mathfrak{S}_{n}$ и, значит, существует бесконечная последовательность (3) классов $\mathbb{Z}$-модулей, которая не стабилизируется на конечном шаге. 


\section{СПИСОК ЦИТИРОВАННОЙ ЛИТЕРАТУРЫ}

[1] О. Ю. Шмидт, "Группы, все подгруппы которых специальные”, Матем. сб., 31 (1924), 366-372.

[2] Л. А. Шеметков, “О. Ю. Шмидт и конечные группы”, Укр. матем. журн., 23:5 (1971), 586-590.

[3] В. С. Монахов, "Подгруппы Шмидта, их существование и некоторые приложения", Украінський математичний конгрес, 2001, Алгебра і теорія чисел, Ин-т матем. НАН Украины, Киів, 2002, 81-90.

[4] Ф. Каш, Модули и кольца, Мир, М., 1981.

[5] И. Ламбек, Колъц,а и модули, Мир, М., 1971.

[6] Общая алгебра, т. 1, Справочная математическая библиотека, ред. Л. А. Скорняков, Наука, М., 1990; т. 2, Справочная математическая библиотека, ред. Л. А. Скорняков, Наука, М., 1991.

[7] К. Фейс, Алгебра: кольц, модули и категории, т. 1, Мир, М., 1977; т. 2, Мир, М., 1979.

[8] Н. Бурбаки, Коммутативная алгебра, Мир, М., 1971.

[9] А.Г. Курош, Лекиии по общей алгебре, Физматгиз, М., 1962.

\section{В. А. Ведерников}

Московский городской педагогический университет

E-mail: vavedernikov@mail.ru

Н. В. Якубовский

Московский педагогический государственный университет
Поступило

27.12 .2005

Исправленный вариант

04.04 .2008 